\title{
Temperature Dependence of Photocurrents Produced by X and Gamma Rays in Silicon Radiation Detectors
}

\author{
Karl Scharf and Robert K. Mohr* \\ Institute for Basic Standards, National Bureau of Standards, Washington, D.C. 20234
}

(August 9, 1971)

\begin{abstract}
The temperature dependence of de photocurrents produced by $\mathrm{x}$ and gamma rays in silicon radiation detectors of the $n-p, p-i-n$, and surface-barrier type was investigated in a temperature range between 20 and $50{ }^{\circ} \mathrm{C}$. Photodiode photocurrents, assumed as being equal to the generated currents $I_{g}$, showed a positive temperature dependence in all detectors investigated. Their temperature coefficient at $25{ }^{\circ} \mathrm{C}$ varied between +0.004 per ${ }^{\circ} \mathrm{C}$ and +0.002 per ${ }^{\circ} \mathrm{C}$. The temperature dependence of short-circuit currents $I_{\mathrm{sc}}$ measured by a compensation method, was positive and nearly linear for $n-p$ type detectors but nonlinear and negative for $p-i-n$ and surface-barrier type detectors. It is shown, that this different behavior of individual detectors is due to the influence of the strongly temperaturedependent junction current $I_{j}$ which under the short-circuit mode is drained off the generated current $I_{g}$. The junction current is a function of the internal series resistance $R_{s}$ and the junction resistance $R_{j}$ of the irradiated detector $\left(I_{j}=I_{\mathrm{sc}} R_{s} / R_{j}\right)$. With increasing resistance ratio $R_{s} / R_{j}$, the current ratio $\boldsymbol{I}_{j} / \boldsymbol{I}_{g}$ increases and the temperature coefficient $\alpha_{\mathrm{sc}}$ of the short-circuit current decreases. Temperature coefficients $\left(\alpha_{\mathrm{sc}}\right)_{25}$ measured in the different detectors at $25{ }^{\circ} \mathrm{C}$ and a current density $6 \times 10^{-7} \mathrm{~A} / \mathrm{cm}^{2}$ decreased with increasing resistance ratio from +0.004 per ${ }^{\circ} \mathrm{C}$ to -0.005 per ${ }^{\circ} \mathrm{C}$. Resistance ratios $R_{s} / R_{j}$ of the detectors investigated ranged between 0.01 and 0.24 approximately. Thus, $\left(\alpha_{\mathrm{sc}}\right)_{25}$ measured in an individual detector can be changed by changing its effective series resistance. A decrease of $\left(\alpha_{\mathrm{sc}}\right)_{25}$ with increasing $I_{\mathrm{sc}}$ was observed in detectors with larger resistance ratios. This was apparently due to the voltage dependence of $R_{j}$ at higher junction voltages produced by larger short-circuit currents.
\end{abstract}

Key words: Gamma rays; photocurrents; radiation; radiation detector; radiation dosimetry; silicon radiation detector; temperature dependence; $\mathrm{x}$ rays.

\section{Introduction}

Silicon radiation detectors operated as direct-current measuring devices can conveniently be used for measurements of exposure rates of $\mathbf{x}$ and gamma rays larger than approximately $0.1 \mathrm{R} / \mathrm{min}[1,2] .{ }^{1}$ Under this mode of operation, exposure rates are determined by measuring steady-state direct currents produced by irradiation in the detector circuit. These radiation-produced signal currents, in general called here photocurrents, are related to the exposure rate of the respective radiation incident on the detector. Detectors can be used as photodiodes [3] when photocurrents are large compared with the dark current produced without irradiation by an external bias voltage. Otherwise, detectors are operated as photovoltaic cells [4]. In this case no external bias voltage is applied at the detector and the total load current measured under irradiation is the photovoltaic photocurrent. However, its magnitude as well as its temperature dependence are functions of the external load resistance used. It is therefore recom-

*Present address: University of Massachusetts, Amherst, Mass. 01002.

1 Figures in brackets indicate the literature references at the end of this paper mended that exposure rates be determined by measuring the photovoltaic short-circuit current which is proportional to exposure rate over a wide range. Shortcircuit currents can be determined by extrapolating photovoltaic current measurements to zero load resistance [4], but are usually approximated by photovoltaic currents measured at small load resistances [1].

An important problem in exposure rate measurements with dc operated silicon radiation detectors is the temperature dependence of photocurrents, in particular that of short-circuit currents. In previous investigations $[3,4]$, short-circuit currents as well as photodiode photocurrents produced by $\mathrm{x}$ rays in diffused silicon radiation detectors of the diffused $p-n$ junction type were found to increase with increasing temperature varying approximately from 20 to $50{ }^{\circ} \mathrm{C}$. Other authors reported similar measurements with silicon radiation detectors of different types but differed in their findings. For instance, temperatureindependent short-circuit currents were observed in diffused $p-n$ junction [5] and surface-barrier type [1] detectors as well as in $p-i-n$ type detectors with shallow lithium drifts $[5,6]$, but short-circuit currents showing a negative temperature dependence were 
observed in $p-i$ - $n$ type detectors with deep lithium drifts [5]. No definite explanation could be given for such different behavior of individual detectors.

In this paper an investigation is reported of the temperature dependence of short-circuit currents and photodiode photocurrents produced by $\mathrm{x}$ and gamma rays in silicon radiation detectors of different types. An electrical compensation method, described in a previous paper [8], was used for a direct and precise measurement of the short-circuit current. Under this method, the voltage drop produced by the photovoltaic current across the external load resistance is compensated by an applied bias voltage. Then, the apparent load resistance and the detector input voltage under irradiation are equal to zero and the current in the external circuit is equal to the short-circuit current. By using this method, it was possible to eliminate any influence of the external load resistance on the temperature dependence of short-circuit currents. In particular, it is shown that differences in the temperature dependence of short-circuit currents observed with detectors of different types can be related to the different values of internal series and junction resistances of individual detectors.

\section{Circuit Equations}

Circuit equations for the direct-current operation of a radiation detector can be derived from the simplified equivalent circuit of a detector shown in figure 1 . The detector is represented by a dc current generator shunted by a rectifying diode of a resistance $R_{j}$,

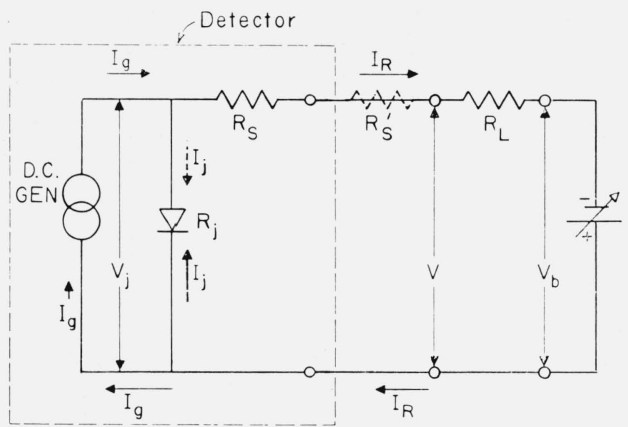

FIGURE 1. Simplified equivalent circuit of an irradiated silicon radiation detector.

called here the junction resistance, and an internal series resistance $R_{s}$. Sometimes it may be desirable to add, as shown, an additional series resistance $R_{s}^{\prime}$ in the circuit as will be discussed below.

The junction resistance $R_{j}$ is a nonohmic resistance. According to the theory of an ideal diode, the voltagedependence of a current $I_{j}$ passing through a diode junction is given by the relation

$$
I_{j}=I_{o}\left[\exp \left(q V_{j} / k T\right)-1\right]
$$

where $V_{j}$ is the voltage across the junction, $I_{j}$ the junction current, $T$ the absolute temperature, $k$ the Boltzman constant, $q$ the electronic charge, and $I_{o}$ the reverse saturation current of the diode. Both $V_{j}$ and $I_{j}$ are assumed to be positive in the forward direction (broken arrow) and negative in the reverse direction (solid arrow) of the rectifying diode junction. The dynamic junction resistance derived from eq (1) as

$$
d V_{j} / d I_{j}=\left(k T / q I_{o}\right) \exp \left(-q V_{j} / k T\right)
$$

is a nonlinear function of the junction voltage $V_{j}$ and decreases or increases with increasing voltage depending on whether $V_{j}$ is in the forward or reverse direction of the junction. However, for voltages $V_{j}<<k T / q$, it may be assumed that $R_{j}$ is a voltageindependent ohmic resistance

$$
R_{j}=(k T) /\left(q I_{o}\right)
$$

The junction resistance $R_{j}$ is strongly temperature dependent because of its dependence on $I_{0}$ which increases exponentially with increasing temperature $T$. At a temperature of $300 \mathrm{~K},(k T) / q$ is equivalent to $26 \mathrm{mV}$ which means that at room temperature eq (3) is applicable only if $V_{j}$ is of the order of a few millivolts.

The internal series resistance $R_{s}$ is assumed here to be an ohmic resistance. It consists of the bulk resistance of the detector, contact resistance, and sheet resistance along the thin contact layer and is usually considered to be negligibly small compared with $\boldsymbol{R}_{j}$. As shown in this investigation, this assumption is apparently not justified in the case of silicon radiation detectors which are made of high-resistivity silicon and are provided with very thin electrical surface contacts.

The bias voltage $V_{b}$ applied to the detector through a load resistance $R_{L}$ (fig. 1 ) is either equal to zero (photovoltaic mode) or is a reverse voltage of finite value (short-circuit mode and photodiode mode). In the latter case $V_{b}$ produces without irradiation a reverse load current, called here the dark current $I_{D}$, which is not shown in figure 1 . When irradiating the detector, a current $I_{g}$ is generated in the reverse direction of the diode junction, causing an increase in the external reverse load current. If $I_{R}$ is the total reverse load current under irradiation (fig. 1) then the photocurrent $I$, defined here as the radiation-produced reverse current signal, is obtained as

$$
I=I_{R}-I_{D} .
$$

In this equation it is assumed that $I, I_{R}$, and $I_{D}$ are reverse currents determined at the voltage $V$ which is applied under irradiation across the detector terminals (fig. 1). Values of $I_{R}$ and $I_{D}$ can be obtained from current-voltage characteristics of the detector measured with and without irradiation. If $V_{b}=0$ or $V=0$, then, as discussed below, $I_{D}$ is either a forward current or is equal to zero and 


$$
I=I_{R}
$$

The generated current $I_{g}$ is proportional to the number of electron-hole pairs produced in the detector by the radiation and collected by the electrical junction field per unit time, and is therefore proportional to the exposure rate. It increases at a small rate when the junction voltage $V_{j}$ increases in the reverse direction, thereby increasing the effective charge collecting volume. However, the generated current $I_{g}$ cannot be directly measured except when operating the detector as a photodiode and using high bias voltages. Thus, exposure rate measurements have to be based on the measurement of the photocurrent $I$ which is usually different from $I_{g}$.

The relation between $I_{g}$ and $I$ can be obtained by determining the relation between $I_{g}$ and $I_{R}$. This can be done by considering the following equation for the voltages $V$ and $V_{j}$ applied under irradiation across the detector terminals and the diode junction respectively. Taking into account the internal series resistance $R_{s}$, then according to the equivalent circuit (fig. 1)

$$
\begin{aligned}
V & =V_{b}-I_{R} R_{L} \\
V_{j} & =V_{b}-I_{R}\left(R_{L}+R_{s}\right)=V-I_{R} R_{s} .
\end{aligned}
$$

Due to the voltage drop produced by $I_{R}$ across $R_{s}$, $V$ and $V_{j}$ are different for a given $I_{R}$. Since under the dc modes $V_{b} \leqslant 0$ and $I_{R}<0$, it follows from eq (6) that for a given $I_{R}, V$ and $V_{j}$ are forward or reverse voltages, i.e., are positive or negative depending on the value of $V_{b}$. In the case of a forward biased junction a forward junction $I_{j}$ is drained off the reverse generated current $I_{g}$ (fig. 1) and

$$
I_{R}=I_{g}-I_{j} .
$$

In the case of a reverse biased junction, a reverse junction current $I_{j}$ is added to $I_{g}$ and

$$
I_{R}=I_{g}+I_{j}
$$

In using eqs (4) to (8) one obtains the following circuit equations for the different dc modes of operation:

(a) Under the photovoltaic mode, $V_{b}=0$. Thus, according to eq (6), $V$ and $V_{j}$ are positive. Therefore, $I_{D}$ and $I_{j}$, which are functions of $V$ and $V_{j}$ respectively, are forward circuits and eqs (5) and (7) are applicable for this mode. Furthermore, the voltage $V_{j}$ is a function of $R_{L}$ and $R_{s}$. This means that $I_{j}$ and consequently the photovoltaic photocurrent (eq (7)) are dependent on the load resistance $R_{L}$, as mentioned above.

(b) Under the short-circuit mode, which is the main subject of this investigation, the short-circuit current is measured by an electrical compensation method. Under this method, a reverse bias voltage, now called the compensation voltage $V_{c}$, is applied under irradiation so that

$$
V_{b}=V_{c}=I_{R} R_{L}
$$

Then the voltage drop produced by $I_{R}$ across the load resistance $R_{L}$ is compensated and according to eq (6)

$$
V=0 \text { and } V_{j}=-I_{R} R_{s}
$$

At $V=0$, the dark current $I_{D}=0$ and $V_{j}$ is positive or a forward voltage because $I_{R}$ is negative. Thus, as under the photovoltaic mode, eqs (5) and (7) can also be applied under the short-circuit mode, noting that $I_{R}$ measured at $V=0$ is called now the short-circuit current, $I_{\mathrm{sc}}$.

Assuming in particular that, at the small junction voltages encountered under this mode, the junction resistance $R_{j}$ may be considered to be an ohmic resistance, then one obtains the junction current as

$$
I_{j}=I_{\mathrm{sc}} R_{s} / R_{j}
$$

and the short-circuit current according to eq (7) as

$$
I_{R} \equiv I_{\mathrm{sc}}=I_{g}\left(1+R_{s} / R_{j}\right)^{-1} .
$$

Compared with the photovoltaic current, the shortcircuit current has the advantage that it is independent of $R_{L}$ and is dependent only on the internal resistances $R_{s}$ and $R_{j}$ which are characteristics unique to each individual detector. The short-circuit current $I_{\mathrm{sc}}$ is smaller than $I_{g}$ but is proportional to $I_{g}$ as long as the ratio $R_{s} / R_{j}$ remains constant. This condition is usually fulfilled when $V_{j}$ remains within the voltage range of constant junction resistance $\boldsymbol{R}_{j}$. According to eq (9), $I_{\mathrm{sc}}$ can be determined by measuring the compensation voltage $V_{c}$ which for a given value of $I_{\text {sc }}$ may be increased by increasing $R_{L}$, thereby increasing the accuracy of exposure rate measurements carried out with silicon radiation detectors.

(c) Under the photodiode mode $\left|V_{b}\right|>\left|I_{R}\right| R_{L}$. In this case the detector voltage $V$ and the dark current $I_{D}$ are in the reverse direction, but depending on whether $|V| \gtrless\left|I_{R}\right| R_{s}$, the junction voltage $V_{j}$ is under irradiation either in the reverse or the forward direction. Thus combining eqs (4), (7), and (8) which are applicable to this mode, one obtains the photodiode photocurrent

$$
I=I_{g}-\left(I_{D} \pm I_{j}\right)
$$

where the minus sign in the bracketed term refers to a reverse biased junction. A distinction is made here between the dark current $I_{D}$ determined by the currentvoltage characteristic of the detector measured without irradiation and the current $I_{j}$ passing through the junction under irradiation. They are related to different voltages $V$ and $V_{j}$ respectively and are therefore of different values as shown below.

It should be noted that in all the above circuit equations, $I_{R}, I$, and $I_{D}$ are values determined as functions of the detector voltage $V$, while $I_{g}$ and $I_{j}$ are related to the corresponding junction voltage $V_{j}$ which, due to the voltage drop across $R_{j}$ is different from $V$ (eq (6)). However, when $I_{D}$ approaches a voltage-independent saturation value at larger reverse detector voltages, then $\left(I_{D}-I_{j}\right) \rightarrow 0$ and $I \rightarrow I_{g}$. Thus, considering photodiode currents at larger reverse detector voltages and neglecting the small voltage dependence of $I_{g}$ 
in the voltage interval between $V$ and $V_{j}$, one may with good approximation assume that for a given voltage $V$ the photodiode current

$$
I=I_{g} .
$$

The influence of the internal detector resistance $R_{s}$ on measured photocurrents is further illustrated in figure 2, showing assumed current-voltage characteristics for a radiation detector having been measured without and with irradiation at constant exposure rate. For convenience, reverse current and voltage values are indicated by positive coordinates. Currents are given by the external characteristics (solid lines) as functions of the measured detector voltage $\mathrm{V}$, and by the internal characteristics (broken lines) as functions of the junction voltage $V_{j}$. For a given current $I_{R}$, the corresponding voltages $V$ and $V_{j}$ differ by the voltage drop of the current across the internal series resistance $R_{s}$, assumed here to be extremely large. For given values of external bias voltage and load resistance, the corresponding values of $I_{R}$ and $V$ are given by the coordinates of the point of intersection between the external characteristic $I_{R}=f(V)$ and the load line crossing the voltage axis at the respective bias voltage $V_{b}$, or the compensation voltage $V_{c}$ in the case of the short-circuit current. In figure $2, I_{g}$ is as an approximation assumed to be voltage-independent and consideration is given only to the behavior of the short-circuit current $I_{\mathrm{sr}}$ and the photodiode current $I$.

When applying at the detector the compensation voltage $V_{c}$, then $V=0$ at the detector and $I_{D}=0$, and the measured current is equal to the short-circuit current $I_{\mathrm{sc}}$. But, as discussed above, $I_{\mathrm{sc}}<I_{g}$ because due to the corresponding voltage $V_{j}$, a forward current $I_{j}$ is deducted from $I_{g}$. If a reverse bias voltage $\left|V_{b}\right|>$ $\left|V_{c}\right|$ is applied, the detector voltage $V$ will be in the reverse direction. At small reverse voltages, $V_{j}$ and $I_{j}$ are in the forward direction but decrease and change into the reverse direction with increasing reverse voltage $V$. Then the respective photodiode photocurrent $I$ is obtained from the external characteristics as the difference between $I_{R}$ and $I_{D}$ at the voltage $V$. The generated current $I_{g}$ is read from the internal characteristics as the difference between the respective current $I_{R}$ and the junction current $I_{j}$ at the voltage $V_{j}$ corresponding to $V$. As long as the dark current $I_{D}$ does not achieve saturation, $I_{D}>I_{j}$, and consequently $I<I_{g}$. It is obvious from figure 2 that $V_{j}$ and $I_{j}$ cannot be obtained from the measured external characteristics unless $R_{s}$ is known and that is usually not the case.

\section{Experimental Procedure}

Most of the detectors investigated were commercially available silicon radiation detectors. Measurements reported here were carried out with two different $n-p$ type detectors, two $p-i-n$ type detectors and one surface-barrier type detector. The $n-p$ type detectors

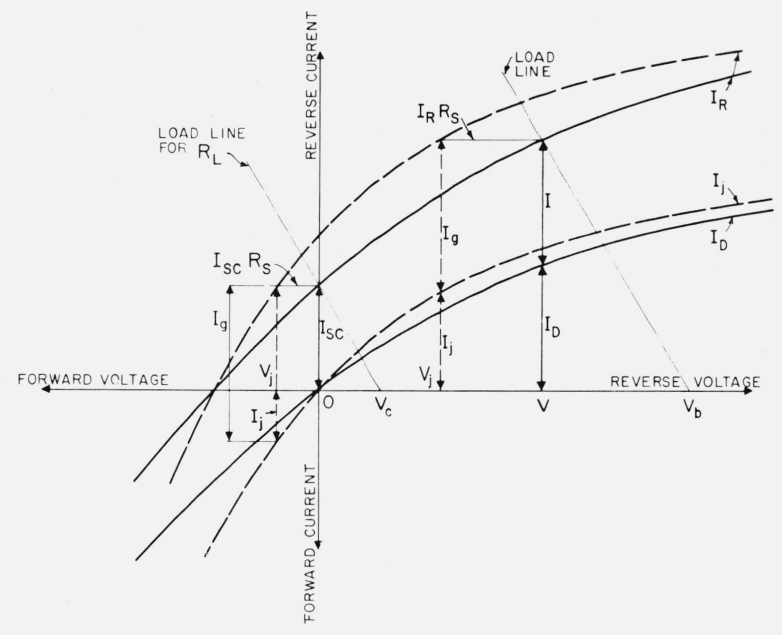

FIGURE 2. Hypothetical current-voltage characteristics of a radiation detector showing external load currents measured with and without irradiation as functions of detector voltage $\mathrm{V}$ (solid lines) and junction voltage $\mathrm{V}_{j}$ (broken lines) respectively.

with surface areas of $2.1 \mathrm{~cm}^{2}$ and $0.3 \mathrm{~cm}^{2}$ respectively were of the encapsulated type with thin protective aluminum coatings on the surfaces. One of the $p-i-n$ detectors, hereafter referred to as $p-i-n$ detector (No. 1) was a lithium-drifted detector with a passivated surface and a specified depletion depth of $2 \mathrm{~mm}$. The other $p-i-n$ detector, hereafter referred to as $p-i-n$ detector (No. 2) was a lithium-drifted detector with a specified depletion depth of $1 \mathrm{~mm}$. The surfacebarrier type detector was a ceramic ring-mounted goldsilicon detector disc placed between pressure contacts. The surface-barrier detector was made of $n$-type silicon of a high unspecified resistivity. All the other detectors were made of $1000 \Omega$-cm $p$-type silicon.

For measurements with $\mathrm{x}$ rays, detectors were mounted in a metallic vacuum-tight container ${ }^{2}$ which was placed inside a thermostatically controlled drying oven (fig. 3). The radiation beam first passed through the nonmetallic oven wall before entering and leaving the container through thin aluminum windows. A lead diaphragm was placed over the entrance window for collimation of the beam. Inside the container the detector was held by a metallic ring mounted on a Bakelite rod. In order to minimize oxidation of detector surfaces, the air pressure in the container was maintained at approximately $10^{-5}$ torr. For measurements with $\gamma$ rays, detectors were placed inside a thermostatically controlled environmental test chamber and exposed to radiation at atmospheric pressure. In both cases the stabilized temperature of the detector was measured with a thermistor-type telethermometer with its probe attached to the metal casing of the detector. All detectors were covered with light-tight aluminum covers of electronic equilibrium thickness [9].

Radiation sources were, if not otherwise stated, a $250 \mathrm{kV}$ tungsten target $\mathrm{x}$-ray tube operated by a stabi-

\footnotetext{
$[3]$
}

${ }^{2}$ The metallic container was originally designed in connection with a previous investigation 


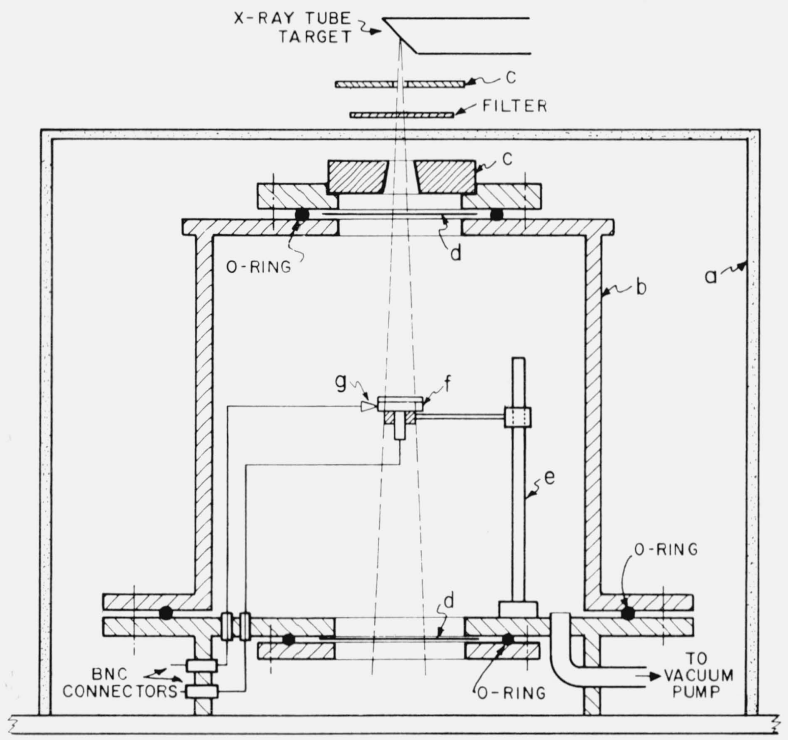

FIGURE 3. Mounting of radiation detector in vacuum-tight metallic container.

(a) Drying oven; (b) metallic container; (c) removable lead diaphragm; (d) thin Al-windows (a) Drying oven; (b) metallic container; (c) removable lead diaphragm; (d) thin Al-windows
for entrance and exit of radiation beam; (e) Bakelite rod with metallic supporting ring; (f) detector; (g) thermistor thermometer probe.

lized constant voltage supply, and a ${ }^{137} \mathrm{Cs} \gamma$-ray source.

Short-circuit currents were measured by a compensation method [8]. Currents observed under the photodiode mode of operation were determined by potentiometric measurement of the voltage drop across a known load resistance.

\section{Temperature Dependence of Photocurrents}

The temperature dependence of short-circuit currents and photodiode photocurrents measured with silicon radiation detectors of different types in a temperature range between 20 and $50{ }^{\circ} \mathrm{C}$ is shown in figure 4. Photocurrents are given in relative values and are normalized to unity at $25{ }^{\circ} \mathrm{C}$. Measurements were made with lightly filtered $100 \mathrm{kV}$ x rays at an approximate exposure rate of $8 \mathrm{R} / \mathrm{min}$. Photodiode photocurrents determined at a bias voltage of 10 $\mathrm{V}$ were obtained from current-voltage characteristics measured at different temperatures.

Short-circuit currents (solid lines) as well as photodiode photocurrents (broken lines) measured with a diffused $n-p$ type detector show a positive temperature dependence and increase with increasing temperature. For the other detectors investigated, photodiode photo-currents also show a positive temperature dependence, but short-circuit currents decrease at varying rates with increasing temperature. The $p-i-n$ detector (No. 2) shows a very small temperature dependence for the short-circuit current. But shortcircuit currents measured with the $p$-i-n detector (No. 1) and the surface-barrier detector show a strongly negative temperature dependence.

Photodiode photocurrents shown in figure 4 were measured at relatively small bias voltages. One may therefore assume that charge collection under the photodiode as well as under the short-circuit mode of operation was mainly achieved by diffusion of minority carriers from the detector base into the depletion region. Thus, the qualitatively different behavior observed under different modes of operation can apparently not be explained by different intrinsic electronic properties of individual detectors, such as mobility and lifetime of minority carriers. An explanation has rather to consider the dependence of the shortcircuit current on the strongly temperature-dependent junction current which is a function of the internal series and junction resistance of each individual detector.

\section{Temperature Coefficients of Photocurrents}

In order to compare the temperature dependence of photocurrents measured with detectors of different types, the temperature coefficient $\alpha$ of the photocurrents at a given temperature is considered here.

In the case of a forward biased junction, as encountered under the photovoltaic and short-circuit mode, the temperature coefficient $\alpha$ can be derived from eq (7). If $\alpha, \beta$, and $\gamma$ are the temperature coefficients of $I, I_{g}$, and $I_{j}$ respectively, defined as

$$
\alpha=(d I / d T) / I ; \quad \beta=\left(d I_{g} / d T\right) / I_{g} ; \gamma=\left(d I_{j} / d T\right) / I_{j}
$$

then by differentiation of eq (7) one obtains

or

$$
\alpha=\left(\beta I_{g}-\gamma I_{j}\right) / \boldsymbol{I}
$$

$$
\alpha \gtrless 0 \text { if }\left(I_{g} / I_{j}\right) \gtrless(\gamma / \beta) \text {. }
$$

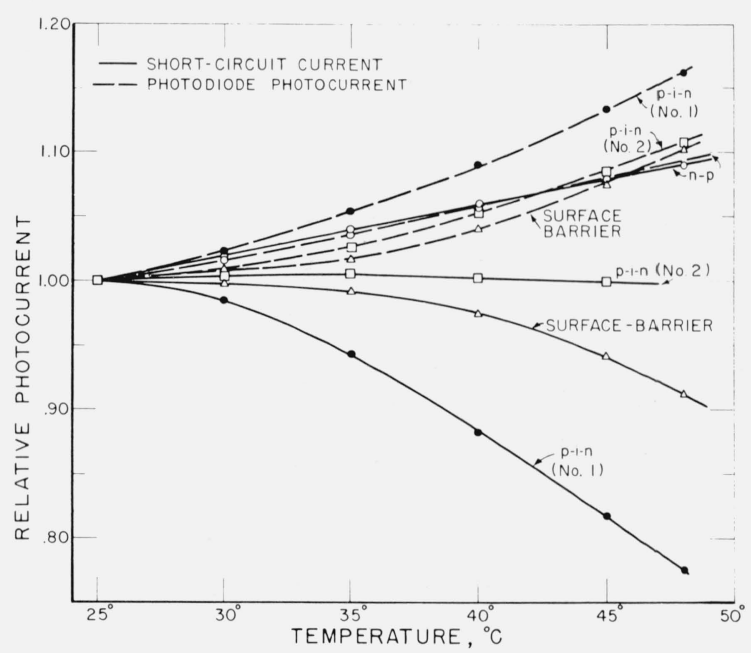

FIGURE 4. Temperature dependence of short-circuit currents (solid lines) and photodiode photocurrents (broken lines) produced by lightly filtered $100 \mathrm{kV} x$ rays in silicon radiation detectors of different types.

The reverse bias voltage used for the measurement of photodiode photocurrents was $V_{b}=10 \mathrm{~V}$. 
The temperature coefficient $\beta$ of $I_{g}$ as derived from photodiode current measurements (fig. 4) is positive, and so is the temperature coefficient of $I_{j}$ which increases rapidly with increasing temperature. However, the temperature coefficient $\alpha$ of photocurrents $I$ can either be positive or negative depending on the value of the ratio $\left(I_{g} / I_{j}\right)$.

Under the photovoltaic mode, $I_{j}$ is a function of the load resistance $R_{L}$ and consequently $\alpha=f\left(R_{L}\right)$ as has been shown in a previous paper [4]. However, under the short-circuit mode, $I_{j}$ is independent of $R_{L}$ and so is the temperature coefficient $\alpha_{\mathrm{sc}}$ of the shortcircuit current. Assuming a constant junction resistance $R_{j}$, one obtains from eqs (11), (12), and (16) the temperature coefficient of the short-circuit current as

$$
\alpha_{\mathrm{sc}}=\beta+\left(R_{s} / R_{j}\right)(\beta-\gamma)
$$

which is dependent only on parameters characteristic of the individual detector. The temperature coefficient $\alpha_{\mathrm{sc}}$ of the short-circuit current is smaller than the temperature coefficient $\beta$ of the generated photocurrent because $\gamma$ is positive and much larger than $\beta$. The contribution of $\gamma$ to the value of $\alpha_{\mathrm{sc}}$ can also be derived as a function of the temperature coefficients of the internal resistances. By differentiation of eq (12) one obtains

$$
\alpha=\beta+\left[R_{s} /\left(R_{j}+R_{s}\right)\right]\left(\delta_{j}-\delta_{s}\right.
$$

if $\delta_{j}$ and $\delta_{s}$ are the temperature coefficients of the junction and series resistance respectively and are defined by relations similar to those given in eq (15). The value of $\delta_{j}$ is negative but $\delta_{s}$ can be positive or negative depending on the structure of the detector. Equation (19) is similar to eq (18) but here $\alpha_{\text {sc }}$ is a function of the ratio of $R_{s}$ and the zero-voltage resistance $\left(R_{s}+R_{j}\right)$ which is equal to the slope $d V / d I_{D}$ at $V=0$ of the external characteristic $I_{D}=f(V)$ shown in figure 2 .

In the case of a reverse biased junction, as in the photodiode mode, eq (16) could be used for values of $I$ measured at small voltages by substituting $\left(I_{D}-I_{j}\right)$ for $I_{j}$. However, for large voltages, one can assume that $I=I_{g}$. In this case the temperature coefficient $\alpha$ of the photodiode photocurrent may be assumed to be equal to the temperature coefficient $\beta$ of the generated current.

\section{Measurement of $\alpha$ as a Function of Different Parameters}

According to equations discussed in the previous section, the temperature coefficient of photocurrents observed in a radiation detector is a function of the junction current $I_{j}$ which is determined by the voltage $V_{j}$ existing across the junction under irradiation. In order to verify these relations, the dependence of the temperature coefficient $\alpha$ on different parameters influencing $V_{j}($ eq (6)) was investigated. Measurements were carried out with the same detectors that were

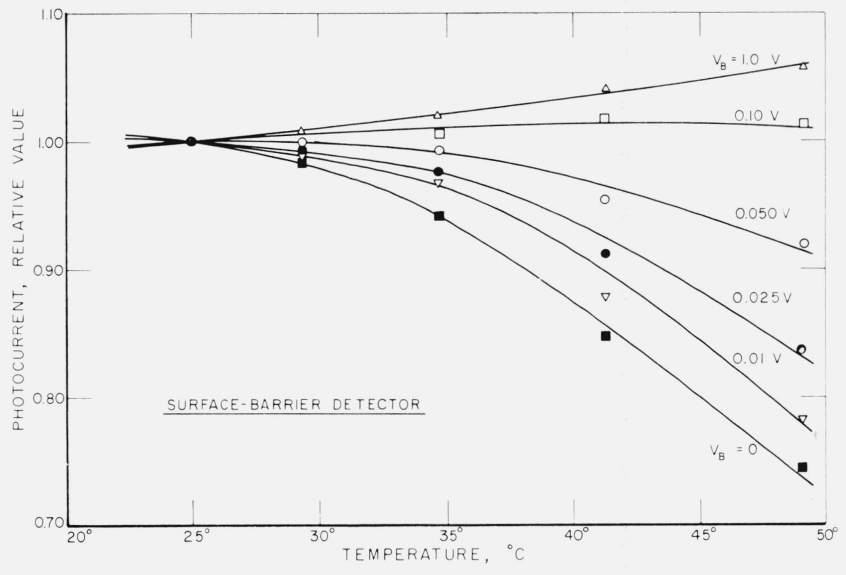

FIGURE 5. Temperature dependence of photocurrents produced by lightly filtered $100 \mathrm{kV} x$ rays in a surface-barrier detector at different detector voltages.

used for measurements shown in figure 4. If not otherwise stated, measurements were carried out with lightly filtered $100 \mathrm{kV}$ x rays.

(a) Detector voltage. The temperature dependence of photodiode photocurrents measured with the surfacebarrier type detector at constant exposure rate for different detector voltages is shown in figure 5 . Voltages $V$ existing at the detector under irradiation and corresponding photocurrents were derived from reverse current-voltage characteristics measured at different temperatures with and without irradiation. Photocurrents are given in relative values normalized to unit at $25^{\circ} \mathrm{C}$. The strongly negative temperature dependence of the short-circuit current at $V=0$, changes gradually with increasing $V$ into the positive temperature dependence of the photodiode photocurrent. The temperature coefficient $\alpha_{25}$ at a given voltage is equal to the slope of the respective curve at $25^{\circ} \mathrm{C}$.

Similar measurements made with detectors of other types are summarized in figure 6 , showing $\alpha_{25}$ as a

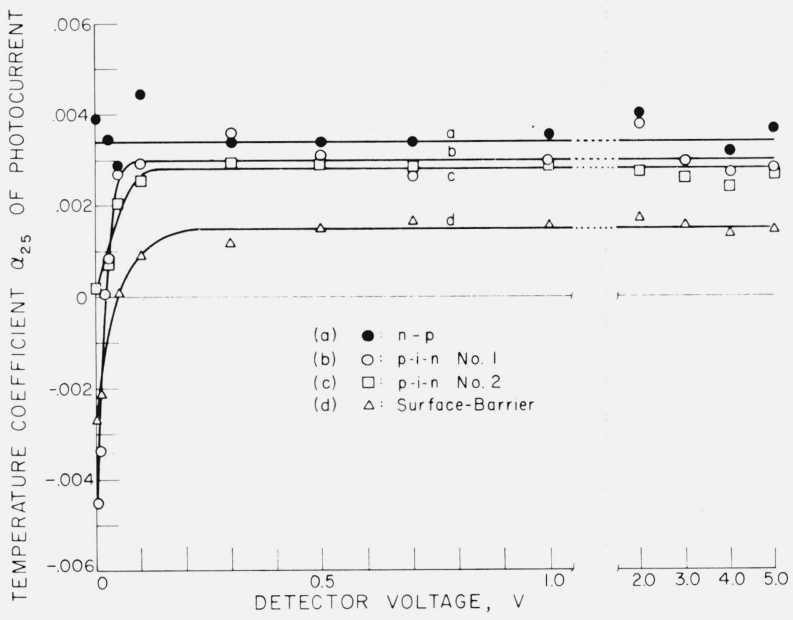

FIGURE 6. Temperature coefficient $\alpha_{25}$ at $25{ }^{\circ} \mathrm{C}$ of photocurrents produced by lightly filtered $100 \mathrm{kV} x$ rays in silicon radiation detectors of different types measured as a function of detector voltage. 
function of $V$. Values of $\alpha_{25}$ for short-circuit currents $(V=0)$ are either positive or negative but change with increasing $V$ into positive values becoming approximately constant at higher voltages. For the diffused $n-p$ type detector $\left(2.1 \mathrm{~cm}^{2}\right), \alpha_{25}$ is nearly voltage-independent over the voltage range investigated. Constant values of $\alpha_{25}$ are reached at relatively small voltages of the order of one volt for all detectors. Apparently at this point $I=I_{g}$ and $\alpha_{25}$ becomes equal to the temperature coefficient of $\beta_{25}$ of the generated photocurrent $I_{g}$. These "plateau" values of $\alpha_{25}$ are of the same order of magnitude for the different detectors but differ slightly apparently due to differences in electronic properties of individual detectors.

(b) Series resistance. In order to change the effective series resistance of a detector, additional resistances $R_{s}^{\prime}$ were inserted in the circuit as shown in figure 1 . These resistances with a negligible temperature coefficient were not incorporated in the detector and were always kept at room temperature. Then photocurrents measured at $V=0$ can be assumed to be approximately equal to the short-circuit current $I_{\mathrm{sc}}^{\prime}$ of the respective detector having an internal resistance $\left(R_{s}+R_{s}^{\prime}\right)$.

The temperature dependence of $I_{s c}^{\prime}$ measured with the diffused $n-p$ type detector at constant exposure rate is shown in figure 7 as a 'function of $R_{s}^{\prime}$. Photocurrents are again given in relative values and the slope of each curve at $25^{\circ} \mathrm{C}$ is the temperature coeffi. cient of $I_{\mathrm{sc}}^{\prime}$ measured with the respective additional resistance $R_{s}^{\prime}$. For $R_{s}^{\prime}=0$, the temperature coefficient of $I_{\mathrm{sc}}^{\prime}$ is positive and decreases with increasing $R_{s}^{\prime}$ changing to negative values at larger $R_{s}^{\prime}$. This behavior is in agreement with eq (17) because at constant

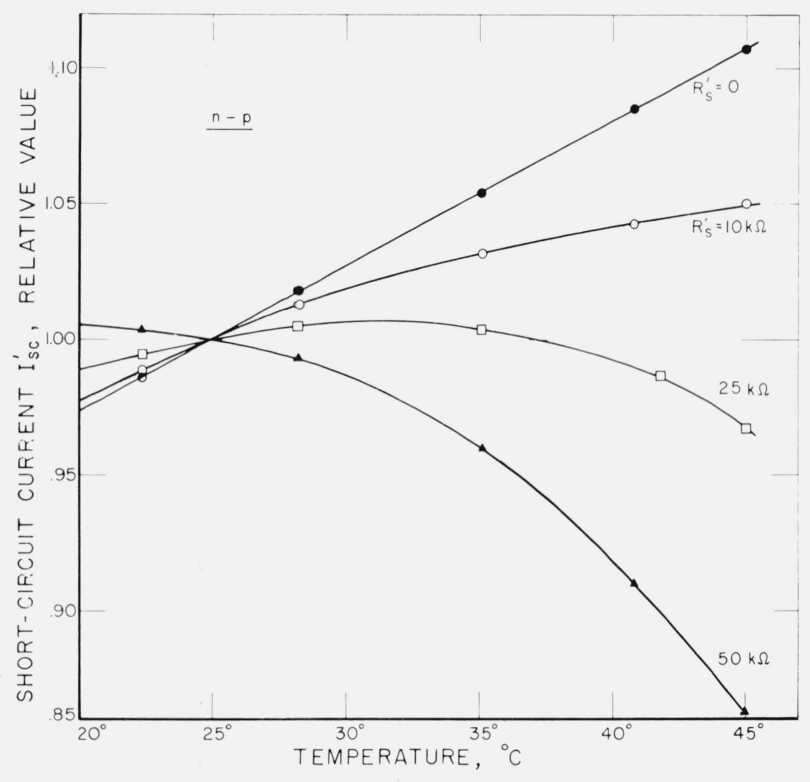

FIgURE 7. Temperature dependence of short-circuit currents $\mathrm{I}_{\mathrm{sc}}^{\prime}$ produced by lightly filtered $100 \mathrm{kV} x$ rays in a diffused silicon $\mathrm{n}-\mathrm{p}$ type detector, measured with different additional series resistances $R_{s}^{\prime}$.

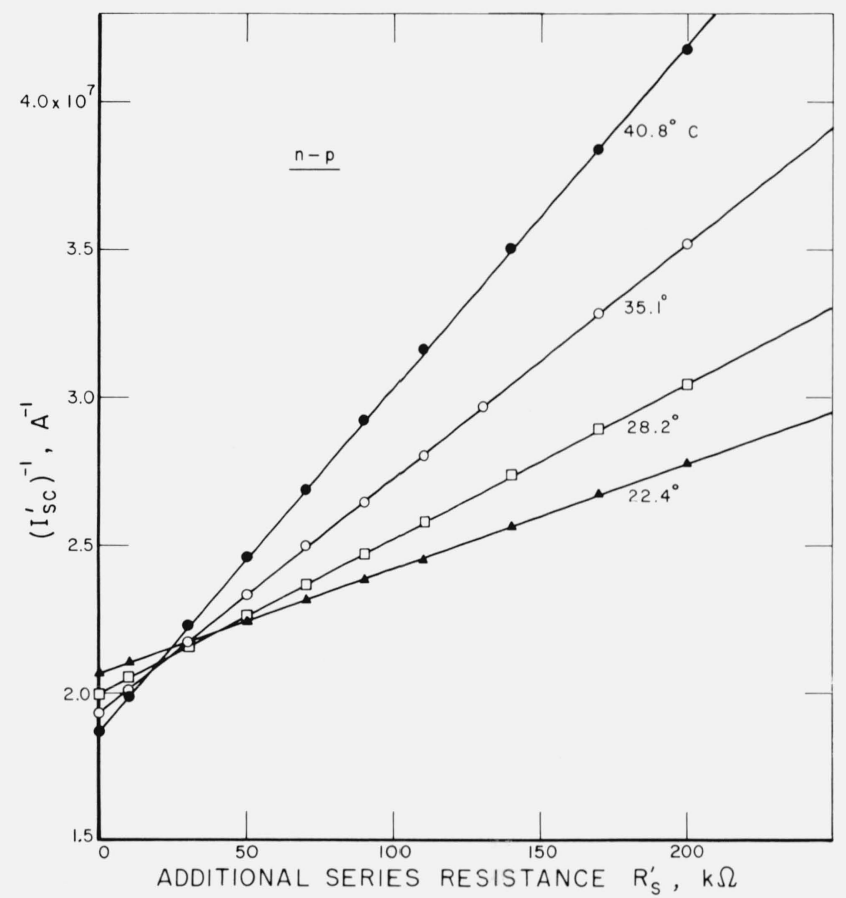

FIGURE 8. Dependence of $\left(\mathrm{I}_{\mathrm{sc}}^{\prime}\right)^{-1}$ on additional series resistance $\mathrm{R}_{\mathrm{s}}^{\prime}$ at different temperatures derived from measurements with a diffused silicon $\mathrm{n}$-p type detector.

exposure rate, i.e., constant $I_{g}$, and constant voltage $V=0$, the junction voltage increases with increasing $R_{s}^{\prime}$ thereby increasing $I_{j}$ and decreasing the ratio $I_{g} / I_{j}$ The temperature dependence of $I_{\mathrm{sc}}^{\prime}$ is similar to that of photovoltaic currents measured with different load resistances [4].

The short-circuit current of the $n-p$ type detector can be made nearly temperature-independent within a certain temperature range by adding a suitable series resistance $R_{s}^{\prime}$ as shown in figure 7. This optimum resistance can conveniently be determined by using a relation derived from eq (12):

$$
\left(1 / I_{s c}^{\prime}\right)=\left(R_{j}+R_{s}+R_{s}^{\prime}\right) /\left(I_{g} R_{j}\right)=\left(1 / I_{\mathrm{sc}}\right)+R_{s}^{\prime} /\left(I_{g} R_{j}\right)
$$

where $I_{\mathrm{sc}}^{\prime}$ and $I_{\mathrm{sc}}$ are the short-circuit currents meas ured with and without an additional resistance $R_{s}^{\prime}$.

According to eq (20), at constant exposure rate and constant temperature $\left(I_{\mathrm{sc}}^{\prime}\right)^{-1}$ is a linear function of $R_{s}^{\prime}$ provided that $R_{j}$ remains constant. Graphs of $\left(I_{\mathrm{sc}}^{\prime}\right)^{-1}$ as a function of $R_{s}^{\prime}$ which were derived from measurements shown in figure 7 are given in figure 8 . Graphs are linear for all temperatures indicating that over the resistance range investigated the junction resistance $R_{j}$ remains constant at each temperature. The change from a positive to a negative temperature dependence of $I_{\mathrm{sc}}^{\prime}$ with increasing $R_{s}^{\prime}$ is indicated by the crossing of the lines. They do not cross at a single point, but points of intersection are spread over a 
small range of resistances $R_{s}^{\prime}$ for which the temperature dependence of $I_{s c}$ is very small.

Similar measurements were carried out with the other detectors with qualitatively similar results. With increasing $R_{s}^{\prime}, \alpha_{25}$ decreases and becomes more negative. Graphs determined for the surface-barrier type detector shown in figure 9 indicate this change in temperature dependence of $I_{\mathrm{sc}}^{\prime}$ but do not show an intersection because at $R_{\mathrm{s}}^{\prime}=0,\left(\alpha_{\mathrm{sc}}\right)_{25}$ is already negative. At lower temperatures, these graphs are nonlinear indicating that the junction resistance $R_{j}$ decreases with increasing $R_{s}^{\prime}$. This is apparently due to an increasing voltage drop across the series resistance which makes the junction voltage so large that $R_{j}$ becomes voltage-dependent and eq (3) is not applicable any more. Linearity of graphs at higher temperatures can apparently be explained by a decrease in junction voltage.

Similar observations were made with the $p-i-n$ type detector (No. 1). For the $p-i-n$ type detector (No. 2) irradiated with heavily filtered $100 \mathrm{kV} \times$ rays at a low exposure rate, graphs of $\left(I_{\mathrm{sc}}^{\prime}\right)^{-1}$ versus $R_{s}^{\prime}$ are linear (fig. 10), but become nonlinear when the detector is irradiated with lightly filtered $240 \mathrm{kV}$ x rays (fig. 11) at a high exposure rate. The explanation given above for the nonlinear cases is supported by measurements discussed in the following section.

(c) Short-circuit current. Temperature coefficients $\left(\alpha_{\mathrm{sc}}\right)_{25}$ of short-circuit currents measured at $25{ }^{\circ} \mathrm{C}$ with detectors of different type and size at different

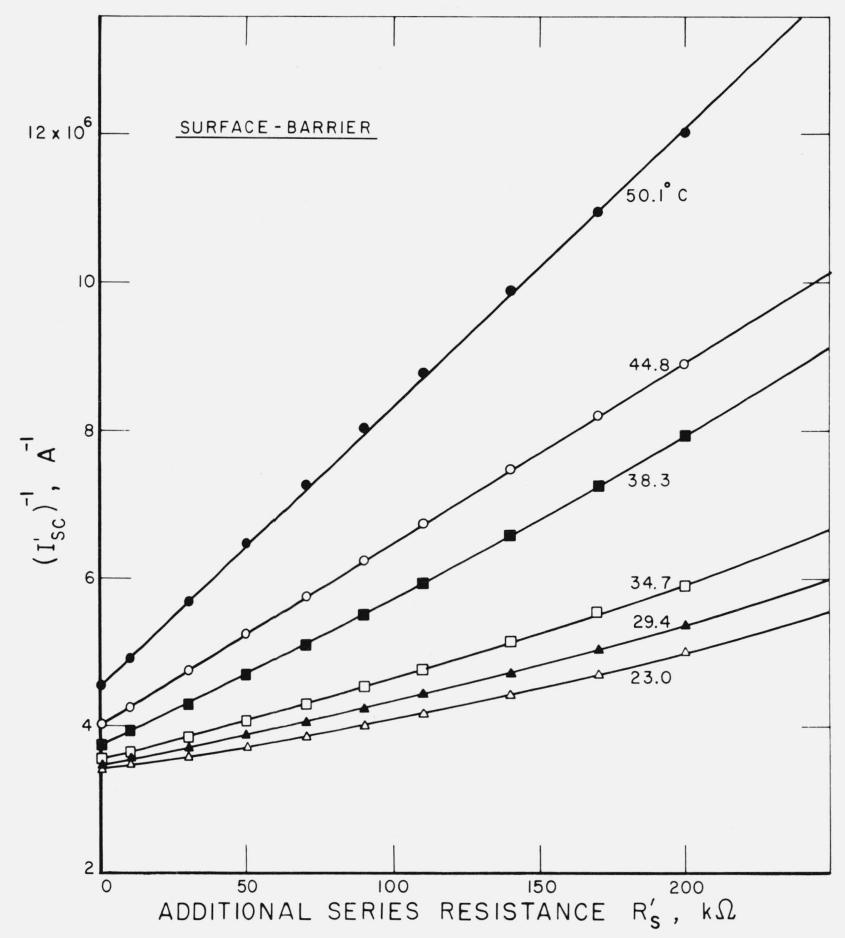

FiguRE 9. Dependence of $\left(\mathrm{I}_{\mathrm{sc}}^{\prime}\right)^{-1}$ on additional series resistance $\mathrm{R}_{\mathrm{s}}^{\prime}$ at different temperatures, measured in a silicon surface-barrier type detector.

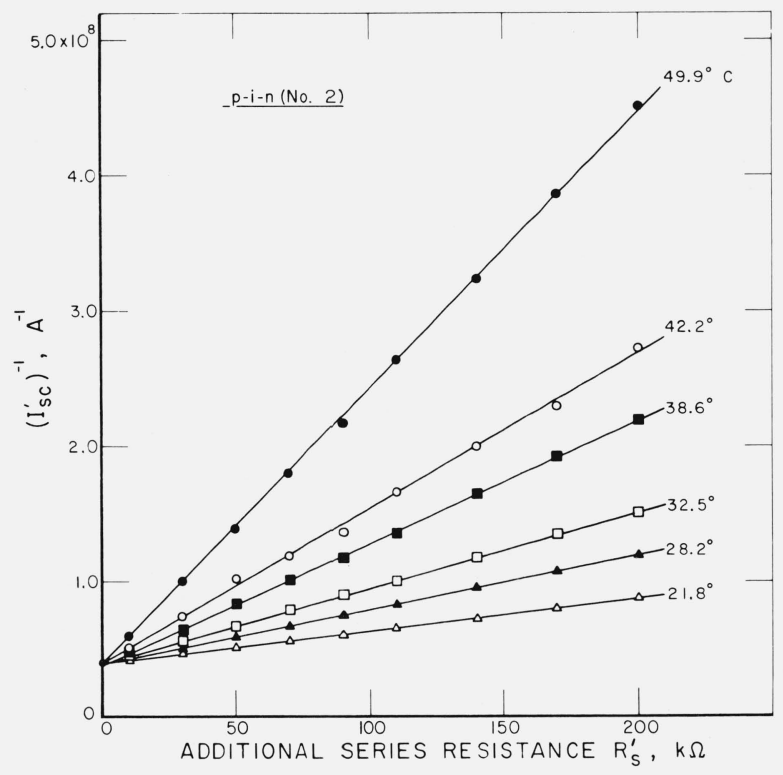

Figure 10. Dependence of $\left(\mathbf{I}_{\mathrm{sc}}^{\prime}\right)^{-1}$ on additional series resistance $\mathbf{R}_{\mathrm{s}}^{\prime}$ measured with p-i-n type detector No. 2, using heavily filtered 100 $k V$ x rays at an approximate exposure rate of $0.6 \mathrm{R} / \mathrm{min}$.

current levels are shown in figure 12. In order to make measurements for detectors of different areas more comparable, $\left(\alpha_{\mathrm{sc}}\right)_{25}$ is shown as a function of shortcircuit current density, considering that in the case of a fully irradiated detector, $I_{g}$ and $I_{\mathrm{sc}}$, as well as $I_{j}$ are proportional to the detector area. Short-circuit currents changing by about three orders of magnitude were produced by $\mathrm{x}$ rays obtained at different tube voltages and different filtrations. The smallest shortcircuit currents of the order of $10^{-9} \mathrm{~A} / \mathrm{cm}^{2}$ were produced by ${ }^{137} \mathrm{Cs}$ gamma rays. The quality of radiation may be assumed to have only a negligible influence on the temperature dependence of $I_{\mathrm{sc}}$.

Values of $\left(\alpha_{\mathrm{sc}}\right)_{25}$ of $I_{\mathrm{sc}}$ remain approximately constant for the diffused $n-p$ type detector $\left(0.3 \mathrm{~cm}^{2}\right)$ and change only slightly for the $p$-i-n type detector (No. 2) over the current range investigated. Howe $2 r$, for the $p$-i-n type detector (No. 1) and for the surtace-barrier type detector, $\left(\alpha_{\mathrm{sc}}\right)_{25}$ decreases markedly with increasing short circuit current. This negative current dependence of $\left(\alpha_{\mathrm{sc}}\right)_{25}$ can again be explained by the voltage drop of the short-circuit current across a large internal series resistance in the detector. With increasing shortcircuit current, the junction voltage equal to this voltage drop reaches a value for which the junction resistance $R_{j}$ becomes voltage dependent.

(d) Internal detector resistances. The relation between the temperature dependence of photocurrents produced in silicon radiation detectors and their internal series and junction resistance has in a qualitative way been shown by the measurements discussed in the previous sections. A quantitative relation between these resistances and the temperature coefficient $\left(\alpha_{\mathrm{sc}}\right)_{25}$ of short-circuit currents can be obtained by determining the resistance ratio $R_{s} / R_{i}$ and the zero- 


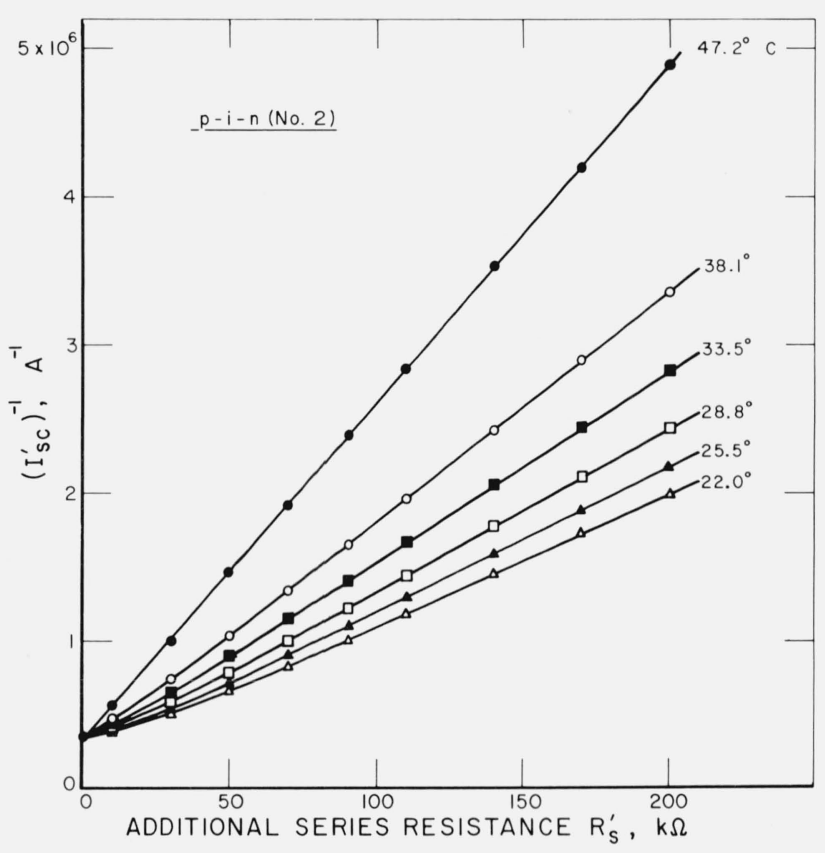

FiguRE 11. Dependence of $\left(\mathrm{I}_{\mathrm{sc}}^{\prime}\right)^{-1}$ on additional series resistance $\mathrm{R}_{\mathrm{s}}^{\prime}$ measured with $\mathrm{p}$-i-n type detector (No. 2), using lightly filtered 240 $k V x$ rays at an approximate exposure rate of $65 \mathrm{R} / \mathrm{min}$.

voltage resistance $\left(R_{s}+R_{j}\right)$ of a detector from its measured current-voltage characteristics. From these values, the series resistance $R_{s}$ and the junction resistance $R_{j}$ can then be calculated. According to eq (12), $R_{s} / R_{j}$ can be obtained from the ratio $I_{g} / I_{\text {sc }}$ where $I_{\text {sc }}$ is the measured short-circuit current and $I_{g}$ is the generated photocurrent at $V=0$ which is derived from photodiode photocurrents measured at detector voltages of a few volts by extrapolation to zero detectorvoltage. This extrapolation was based on the measured voltage-dependence of $I_{g}$ which for all detectors investigated was found to satisfy the relation [3]

$$
\left(I_{g}\right)_{V}=a+b\left(V+V_{0}\right)^{1 / 2}
$$

where $\left(I_{g}\right)_{V}$ is the generated current at the detector voltage $V, a$ and $b$ are constants, and $V_{0}$ is the builtin potential difference across the junction, assumed here as an approximation to be $V_{0}=0.5 \mathrm{~V}$.

Resistance values determined in this way for the different detectors investigated, and the respective temperature coefficients of short-circuit currents are given in table 1 . Values of $\left(\alpha_{\mathrm{sc}}\right)_{25}$ at a short-circuit density of approximately $6 \times 10^{-7} \mathrm{~A} / \mathrm{cm}^{2}$ were taken from curves shown in figure 12. The high series resistances of the surface-barrier type and the $p-i-n$ type detector (No. 1) may be due to surface oxide layers or nonohmic contacts on these detectors. The relative low junction resistances of both $p-i-n$ type detectors may be explained by an inhomogeneous distribution of lithium ions forming low-resistivity channels shunting the junction field region. It should be noted that measured values of $R_{s}$ and $R_{j}$ are effective values which are determined by a combination of different series and shunt resistances not specifically considered here.

According to table 1 , and as shown in figure $13,\left(\alpha_{\mathrm{sc}}\right)_{25}$ is not changing in a way similar to that of the individual values of $R_{s}$ or $R_{j}$, but decreases monotonically with the increasing ratio of these resistances. This result is in agreement with eq (18), though according to this equation a linear dependence of $\left(\alpha_{\mathrm{sc}}\right)_{25}$ on $R_{s} / R_{j}$ may have been expected. The nonlinearity of the relation between $\left(\alpha_{\mathrm{sc}}\right)_{25}$ and the resistance ratios as shown in figure 13, may be due to differing temperature coefficients $\beta$ and $\gamma$ of the currents $I_{g}$ and $I_{j}$ in individual detectors and to the voltage dependence of $R_{j}$ at higher junction voltages. Junction voltages $\left(V_{j}=I_{\mathrm{sc}} R_{s}\right)$ calculated for the different detectors investigated are shown in table 1 . At the given current level, the junction voltages $V_{j}$ in the $n-p$ type detector and the $p$-i-n type detector (No. 2) are rather small. However, for the $p$-i-n type detector (No. 1) and the surface-barrier type detector, values of $V_{j}$ are much higher and in such a range that $R_{j}$ and $R_{s} / R_{j}$ may be assumed to be voltage dependent. This confirms the explanation given earlier for the current dependence of $\left(\alpha_{\mathrm{sc}}\right)_{25}$ observed with these detectors. It should be noted that in such cases, values of $R_{j}$ determining the zero voltage resistance $\left(R_{s}+R_{j}\right)$ and those determining the current ratios $I_{g} / I_{\mathrm{sc}}$ are different. Thus, the "true" values of the zero-voltage junction resistances $R_{j}$ may be larger, and the values of the corresponding series resistances $R_{s}$ and resistance ratios $R_{s} / R_{j}$ may be smaller than those given in table 1 and used in figure 13 .

\section{Exposure Rate Dependence of $\boldsymbol{I}_{\mathrm{sc}}$}

The value of the temperature coefficient of the shortcircuit current $I_{\mathrm{sc}}$ (eq (18)) has been derived from eq (12) showing the dependence of $I_{\mathrm{sc}}$ on the resistance ratio $R_{s} / R_{j}$. Hence, any voltage dependence of $R_{j}$

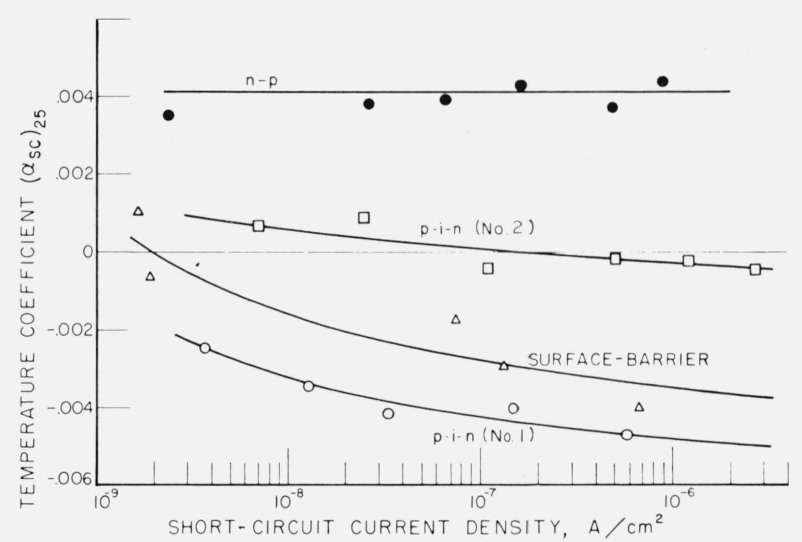

Figure 12. Temperature coefficient $\left(\alpha_{\mathrm{sc}}\right)_{25}$ at $25{ }^{\circ} \mathrm{C}$ of short-circuit currents measured with detectors of different types as a function of short-circuit current density. 
TABLE 1. Temperature coefficients $\left(\alpha_{\mathrm{sc}}\right)_{25}$ of shortcircuit currents measured in silicon radiation detectors at $25{ }^{\circ} \mathrm{C}$ at a current density of $6 \times 10^{-7}$ $\mathrm{A} / \mathrm{cm}^{2}$, and detector data

\begin{tabular}{|c|c|c|c|c|}
\hline \multirow{2}{*}{ Item } & \multicolumn{4}{|c|}{ Detector type } \\
\hline & $n-p$ & $\begin{array}{c}p-i-n \\
\text { (No. 2) }\end{array}$ & $\begin{array}{c}\text { Surface } \\
\text { B. }\end{array}$ & $\begin{array}{c}p-i-n \\
\text { (No. 1) }\end{array}$ \\
\hline$\left(\alpha_{\mathrm{se}}\right)_{25} \ldots \ldots\left({ }^{\circ} \mathrm{C}\right)^{-1} \ldots$ & +0.0040 & -0.0002 & -0.0033 & -0.0047 \\
\hline Area $\ldots \ldots \ldots \mathrm{cm}^{2}$. & 0.3 & 1.1 & 2.3 & 2.5 \\
\hline$R_{s} / R_{j} \ldots \ldots \ldots \ldots \ldots$ & 0.011 & 0.044 & 0.092 & 0.245 \\
\hline$\left(R_{j}+R_{s}\right) \ldots \ldots . \mathrm{k} \Omega$ & 830 & 147 & 866 & 61 \\
\hline$R_{j} \ldots \ldots \ldots \ldots . \mathrm{k} \Omega$. & 821 & 141 & 793 & 49 \\
\hline$R_{s} \ldots \ldots \ldots \ldots \mathrm{k} \Omega$. & 9 & 6 & 73 & 12 \\
\hline$V_{j} \ldots \ldots \ldots \ldots m \mathrm{mV} .$. & 1.6 & 4.0 & 100.7 & 18.0 \\
\hline
\end{tabular}

should influence $I_{\mathrm{sc}}$ as well as $\left(\alpha_{\mathrm{sc}}\right)_{25}$. In order to prove this point, measurements of the exposure rate dependence of $I_{\mathrm{sc}}$, were carried out for the different detectors as shown in figures 14 and 15. For small short-circuit currents produced by ${ }^{137} \mathrm{Cs}$ gamma rays, $I_{\mathrm{sc}}$ is a linear function of exposure rate for all detectors. However, for large short-circuit currents produced at high exposure rates of lightly filtered $30 \mathrm{kV}$ $\mathrm{x}$ rays obtained from a beryllium window-type tube, the exposure rate dependence of $I_{\mathrm{sc}}$ is non-linear for detectors which have a high resistance ratio and show a relatively large current dependence of $\left(\alpha_{\mathrm{sc}}\right)_{25}$ (fig. 14). This nonlinearity can again be explained by the increase of $R_{s} / R_{j}$ with increasing exposure rate, i.e., increasing short-circuit current, due to the voltage dependence of $R_{j}$. According to eq (12), $I_{\text {sc }}$ remains proportional to $I_{g}$, i.e., to exposure rate, only as long as the resistance ratio $R_{s} / R_{j}$ remains constant. Shortcircuit currents observed with the $p-i-n$ type detector (No. 2) (fig. 14) show only a slight deviation from a linear exposure rate dependence, but for the $n-p$ type detector $\left(2.1 \mathrm{~cm}^{2}\right)$ which had a resistance ratio of about $0.015, I_{\mathrm{se}}$ is proportional to exposure rate over the whole range investigated (fig. 15).

\section{Summary and Conclusions}

The following conclusions can be drawn from this investigation of the temperature dependence of photocurrents produced by $\mathrm{x}$ and gamma rays in silicon radiation detectors of the diffused $n-p$, lithium drifted $p-i-n$, and surface barrier type in a temperature range between 20 and $50^{\circ} \mathrm{C}$ approximately.

(1) Generated photocurrents derived from photodiode measurements showed an increase with increasing temperature in all detectors investigated. Although there is no complete explanation for this positive temperature dependence it is, at least in part, due to an increase in the effective diffusion length of minority carriers with increasing temperature, as has previously been shown [3].

(2) The temperature dependence of the shortcircuit current was similar to that of the generated current in the diffused $n-p$ type detectors only, but was in a varying degree non-linear and negative for the other detectors. It is shown that this different behavior can be explained by the influence of the strong positive temperature-dependence of the junction current which in the short-circuit mode is deducted from the generated photocurrent. This junction current is a function of the internal series resistence $R_{s}$ and the junction resistarice $R_{j}$ of the respective detector. With increasing resistance ratio $R_{s} / R_{j}$, the junction current increases and the temperature coefficient of the short-circuit current decreases. Assuming a temperature coefficient at $25^{\circ} \mathrm{C}$ of about 10 percent per ${ }^{\circ} \mathrm{C}$ for the junction current and about 0.4 percent per ${ }^{\circ} \mathrm{C}$ for the generated photocurrent, then the temperature coefficient $\left(\alpha_{\mathrm{sc}}\right)_{25}$ of the shortcircuit current should become nogative when the junction current is larger than about 4 percent of the generated photocurrent (eq (16)) or $R_{s}$ is larger than approximately the same fraction of $R_{j}$. These values were confirmed by determining the resistance ratios from the current-voltage characteristics of the individual detectors.

(3) Values of the temperature coefficients $\beta_{25}$ of the generated photocurrent, determined by measurements of photodiode photocurrents, varied approximately between +0.004 per ${ }^{\circ} \mathrm{C}$ and +0.002 per ${ }^{\circ} \mathrm{C}$ for the different detectors (fig. 6). Values of $\left(\alpha_{\mathrm{sc}}\right)_{25}$ for short-circuit currents changed with increasing resistance ratio of individual detectors of different types from about +0.004 per ${ }^{\circ} \mathrm{C}$ to -0.005 per ${ }^{\circ} \mathrm{C}$.

(4) Detectors with larger series resistances showed a current dependence of $\left(\alpha_{\mathrm{sc}}\right)_{25}$ decreasing with increasing short-circuit currents. This can be explained by a decrease of $R_{j}$ at higher forward junction voltages resulting in an increase of the resistance ratio and a corresponding decrease of $\left(\alpha_{\mathrm{sc}}\right)_{25}$. This explanation was confirmed by the measurement of the exposure rate dependence of the short-circuit current which is also a function of the resistance ratio. For

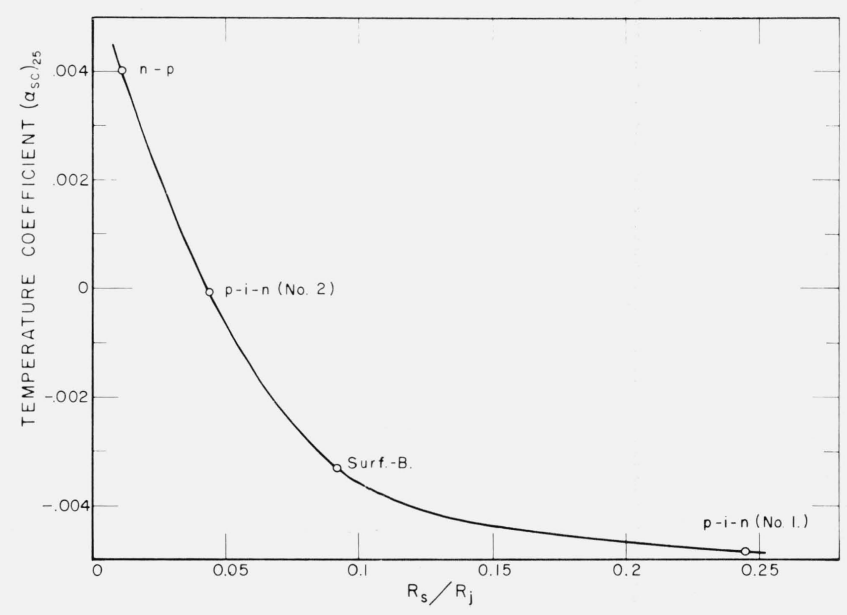

Figure 13. Temperature coefficient $\left(\alpha_{\mathrm{sc}}\right)_{25}$ at $25{ }^{\circ} \mathrm{C}$ and current density $6 \times 10^{-7} \mathrm{~A} / \mathrm{cm}^{2}$ of short-circuit currents measured with detectors of different types as a function of the ratio of their individual series and junction resistance $\mathrm{R}_{\mathrm{s}} / \mathrm{R}_{\mathrm{j}}$. 


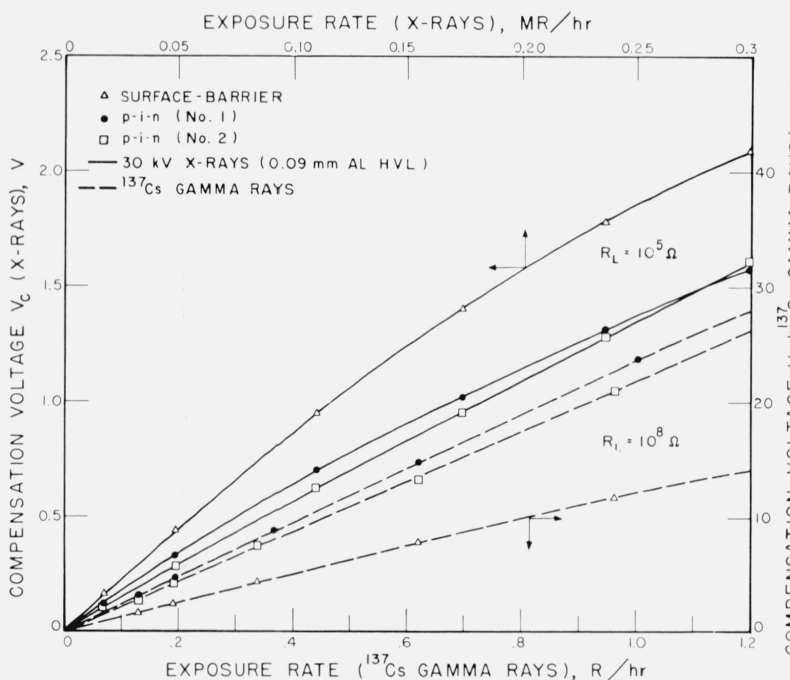

FigURE 14. Exposure rate dependence of compensation voltage, determining the short-circuit currents, as measured with silicon $\mathrm{p}-\mathrm{i}-\mathrm{n}$ and surface-barrier type detectors at high exposure rates of lightly filtered $30 \mathrm{kV} x$ rays and at low exposure rates of ${ }^{137} \mathrm{Cs}$ gamma rays.

detectors with high resistance ratios, the exposure rate dependence is linear at low current levels and becomes non-linear at large currents.

(5) According to the above findings it is possible to change the value of $\left(\alpha_{\mathrm{sc}}\right)_{25}$ by changing the resistance ratio. By adding to a detector an additional

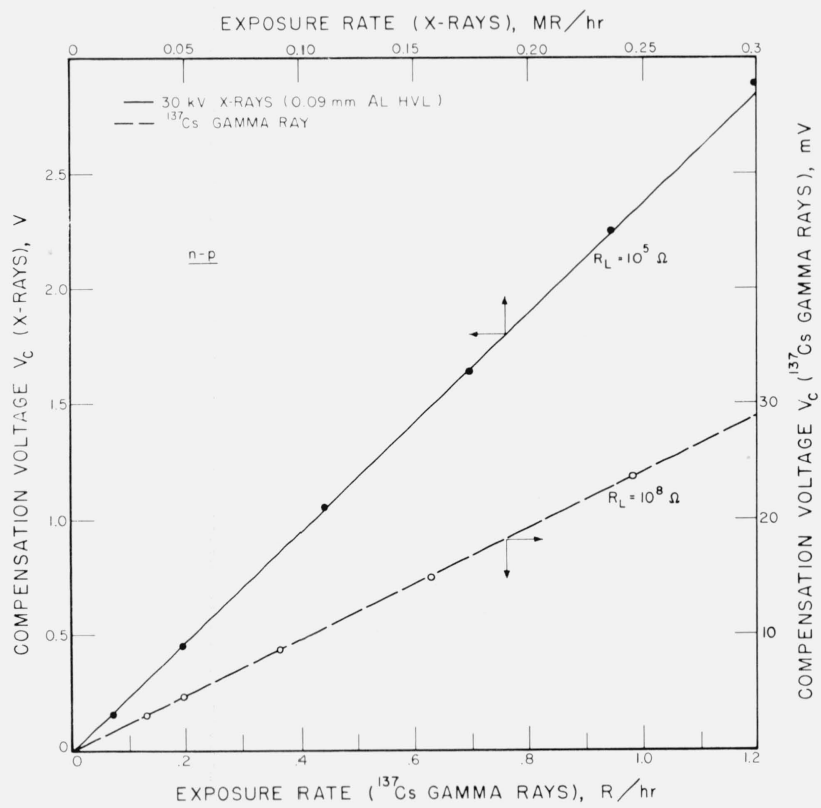

FiguRE 15. Exposure rate dependence of compensation voltage, determining the short-circuit currents as measured with a diffused silicon n-p type detector at high exposure rates of lightly filtered $30 k V \times$ rays and at low exposure rates of ${ }^{137} \mathrm{Cs}$ gamma rays. resistance of small temperature dependence, the short-circuit currents showing a positive $\left(\alpha_{\mathrm{sc}}\right)_{25}$ can be made nearly independent of temperature within a certain temperature range. In the case of a negative $\left(\alpha_{\mathrm{sc}}\right)_{25}$, the additional resistance must be of the thermistor type having a strong negative temperature dependence. However, by using an additional resistance, care must be taken that a linear exposure rate dependence is retained in the range of interest.

(6) By measuring the resistance ratio of an individual detector, its behavior with regard to temperature and exposure rate dependence can be predicted qualitatively.

(7) The diffused $n-p$ type detectors showed the best performance characteristic with regard to temperature and exposure rate dependence of short-circuit currents. Obviously no generalization can be made for the behavior of individual detectors of different types. But the larger resistance ratios observed with the other detectors seem to be typical, being due either to large series resistances as in the case of surface-barrier type detectors, or to relatively small junction resistances as in the case of $p-i-n$ type detectors.

\section{References}

[1] Parker, R. P. and Morley, B. J., Silicon $p-n$ junction surface barrier detectors and their application to the dosimetry of $\mathrm{x}$ - and gamma-ray beams, Proc. Solid State and Chemical Radiation Dosimetry in Medicine and Biology Symp., Vienna, October 3-7, 1966, pp. 167-183 (International Atomic Energy Agency, Vienna, 1967)

[2] Fowler, J. F., Solid state electrical conductivity dosimeters, Chapter 14, in Radiation Dosimetry, 2nd Edition, Eds. F. H. Attix and W. C. Roesch, pp. 291-305 (Academic Press, New York, 1966).

[3] Scharf, K., and Sparrow, J. H., Steady-State response of silicon radiation detectors of the diffused $p-n$ junction type to $\mathrm{x}$ rays. II: Photodiode mode of operation, J. Res. Nat. Bur. Stand. (U.S.), 70A (Phys. and Chem.) No. 2, 181-191 (March-April 1966).

[4] Scharf, K. and Sparrow, J. H., Steady-State response of silicon radiation detectors of the diffused $p-n$ junction type to $\mathrm{x}$ rays. I: Photovoltaic mode of operation. J. Res. Nat. Bur. Stand. (U.S.), 68A (Phys. and Chem.) No. 6. 683-701 (Nov.-Dec. 1964).

[5] Laughlin, J. S., Biological and clinical dosimetry, Bull. Am. Phys. Soc. (Series 2), 11 , No. 6, 4963 (Nov. 1966).

[6] Bailey, N. A., and Kramer, G., The lithium-drifted silicon $p-i-n$ junction as an $\mathrm{x}$-ray and gamma-ray dosimeter, Radiation Research, 22, No. 1, 53-79 (May 1964).

[7] Whelpton, D., and Watson, B. W.. A $p-n$ junction photovoltaic detector for use in radiotherapy, Phys. Med. Biol. 8, No. 1, 33-42 (April 1963).

[8] Scharf. K., Exposure rate measurements of x- and gamma-rays with silicon radiation detectors, Health Phys. 13, No. 6, 575-586 (June 1967).

[9] White, G. N., Measurement of exposure dose, Chapter 5 in Principles of Radiation Dosimetry, pp. 62-64 (John Wiley \& Sons, Inc., New York 1959).

(Paper 75A6-690) 